\title{
Shape Control of CdTe Nanocrystals: Influence of the Solvent Composition and Ligand Effects
}

\author{
Xiaoping Jin, Jürgen Parisi, and Joanna Kolny-Olesiak \\ Energy and Semiconductor Research Laboratory, Department of Physics, Carl von Ossietzky University of Oldenburg, \\ Carl von Ossietzky Straße 9-11, 26111 Oldenburg, Germany \\ Correspondence should be addressed to Joanna Kolny-Olesiak; joanna.kolny@uni-oldenburg.de
}

Received 15 October 2012; Accepted 1 January 2013

Academic Editor: Vijaya Rangari

Copyright (C) 2013 Xiaoping Jin et al. This is an open access article distributed under the Creative Commons Attribution License, which permits unrestricted use, distribution, and reproduction in any medium, provided the original work is properly cited.

CdTe nanocrystals were synthesized by the hot-injection method with a mixture of oleylamine and octadecene as a solvent. The influence of the composition of the solvent and of the injection solution on the shape of CdTe nanoparticles was investigated. Various shapes of CdTe nanocrystals, such as nanodots, nanorods, multipods, and nanowires, could be obtained by changing the reaction conditions. Tuning the reactivity of both the cadmium and the tellurium precursors at the same time was found to be the main reason for the shape control of CdTe nanocrystals in this reaction system. The reactivity of the Cd precursor was controlled by the composition of the solvent, while the activity of the Te precursor could be influenced by using trioctylphosphine and tributylphosphine in the injection solution.

\section{Introduction}

CdTe is a direct semiconductor with a bandgap in the bulk of $1.475 \mathrm{eV}$ and a Bohr exciton radius of $7.3 \mathrm{~nm}$. CdTe nanocrystals attract interest due to their pronounced quantum size effect and optical activity both in the visible and near-infrared spectral regimes. This makes them interesting candidates for a variety of applications, for example, in solar energy conversion [1,2] or biolaballing [3,4]. Thus, size and shape control of CdTe nanocrystals have been a pursued activity for the past few years [5-11]. Various shapes of CdTe have been obtained by different kinds of synthetic procedures. The hot-injection technique especially allows for synthesizing particularly uniform particles and offers a means to control not only the size of the particles, but also their shape. Uniform quasispherical nanocrystals, nanorods, and tetrapods as well as nanowires were grown by this method [10, 12-17].

In the hot-injection method, monomer activity during the nucleation and growth processes plays a key role for shape control of nanocrystals. Several parameters influence the monomer activity, like precursor concentration, precursor activation, and ligands effect. Ligands as an important part in the synthesis of nanocrystals influence the monomer activity in several ways. Yu et al. [12] demonstrated how ligands affect the shape of CdTe nanocrystals in a noncoordinating solvent. In the present study we investigated the influence of ligands and solvents onto the shape control of CdTe nanocrystals growing in a coordinating solvent (oleylamine, (OLAM)). In our previous works we showed that the growth process of CdTe nanocrystals in OLAM and with oleic acid (OA) as stabilizer can be strongly influenced by even small changes in the reaction conditions, which allows for shape control while keeping the principal reagents fixed [15]. Here we investigate further the possibility to control the morphology of CdTe nanocrystals synthesized in OLAM with OA as ligand. A variety of shapes (dots, rods, multipods, and nanowires) have been realized by the hot-injection method by changing the compositions of the solvent and of the injection solution. The resulting materials were characterized by UV-Vis absorption spectroscopy, powder X-ray diffraction (XRD) measurements, and transmission electron microscopy (TEM).

\section{Experimental}

2.1. Chemicals. All chemicals were used as received without further purification: cadmium oxide (CdO, 99\%, 
Te-TOP-OLAM

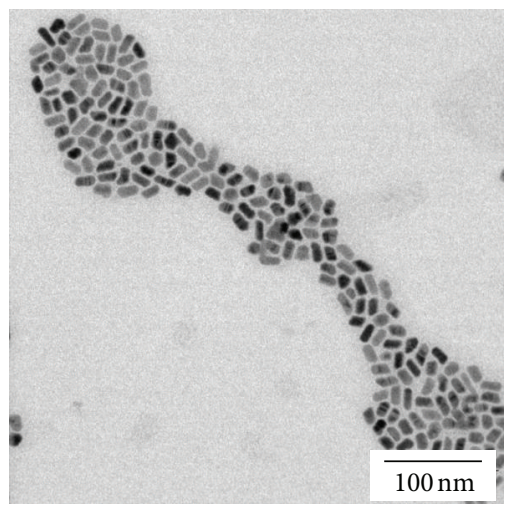

(a)

Te-TOP-ODE

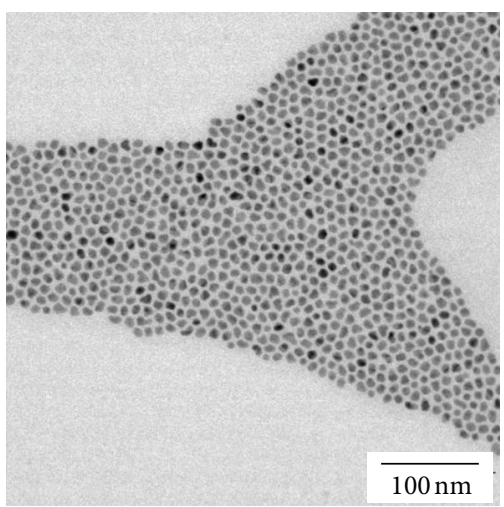

(d)
Te-TOP-TBP-OLAM

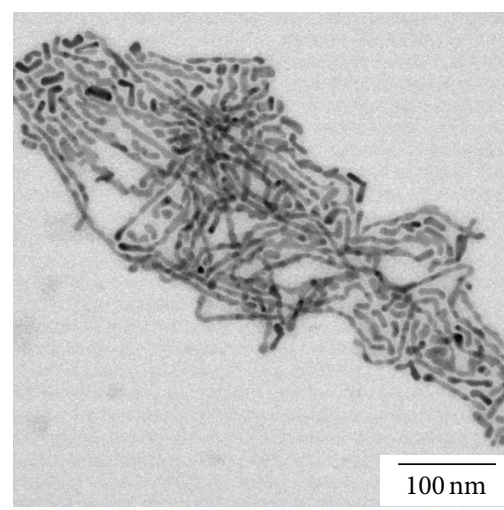

(b)

Te-TOP-TBP-ODE

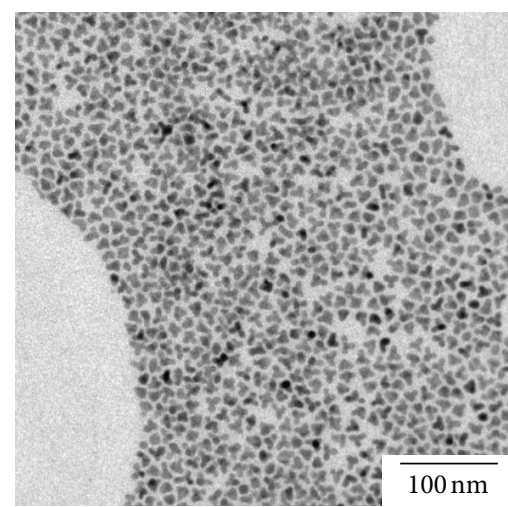

(e)

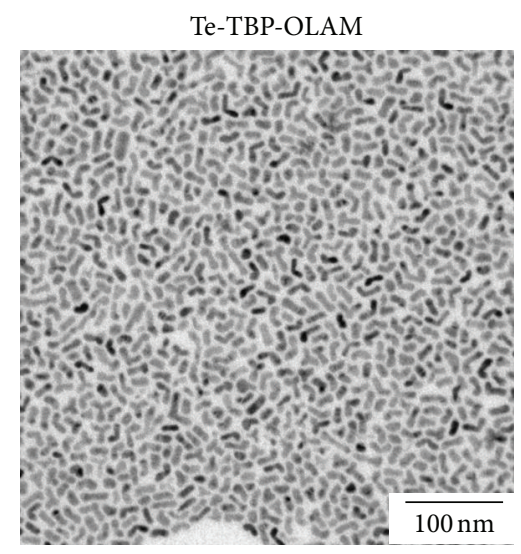

(c)

Te-TBP-ODE

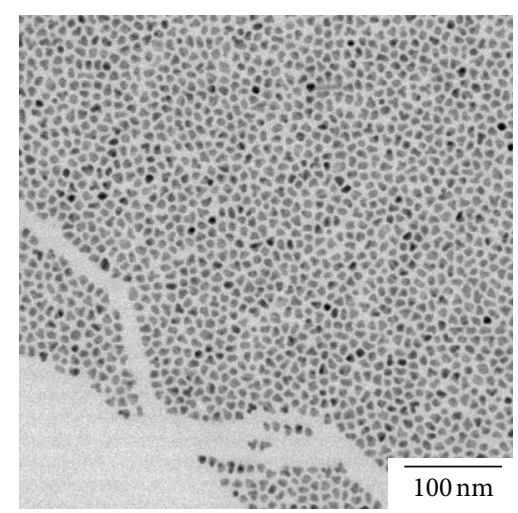

(f)

FIGURE 1: TEM images of CdTe NCs synthesized from different tellurium precursor solutions. (a) Te-TOP-OLAM; (b) Te-TOP-TBP-OLAM; (c) Te-TBP-OLAM; (d) Te-TOP-ODE; (e) Te-TOP-TBP-ODE; (f) Te-TBP-ODE.

Fluka), tri-n-octylphosphine (TOP, 90\%, Aldrich), tri-nbutylphosphine (TBP, 90\%, Aldrich), oleic acid (OA, 90\%, Aldrich), 1-octadecene (ODE, Merck), tellurium powder (Te, purum p., Fluka), and oleylamine (OLAM, approximately C18-content 80-90\%, Acros Organics).

2.2. Te Precursor Preparation. The general procedure for preparing Te precursor solutions is as follows. Te was added to TOP, TBP, or the mixture of them in the glove box. The mixture was further heated to $200^{\circ} \mathrm{C}$ under nitrogen flow till the dissolution of Te. The Te injection solution was obtained by diluting this Te solution with OLAM or ODE (Table 1).

2.3. Synthesis of Colloidal CdTe Nanocrystals with Various Injection Solutions. A mixture of $\mathrm{CdO}(0.1 \mathrm{mmol}), \mathrm{OA}$ $(0.16 \mathrm{~mL})$, and OLAM $(10 \mathrm{~mL})$ was degassed at $100^{\circ} \mathrm{C}$ for 1 hour in a $50 \mathrm{~mL}$ three-necked flask connected to a condenser. The mixture was heated slowly under nitrogen until most of the $\mathrm{CdO}$ decomposed at $\sim 290^{\circ} \mathrm{C}$, then another $0.2 \mathrm{~mL}$ OA was added to form a clear and colourless solution. Te precursor solution (including $0.05 \mathrm{mmol}$ tellurium) was injected quickly at $300^{\circ} \mathrm{C}$. The temperature dropped to $260^{\circ} \mathrm{C}$ and maintained this value throughout the synthesis. All syntheses were stopped after 5 minutes by removing the heating mantle and by rapidly cooling the flask. Aliquots were taken out at different reaction times to monitor the reaction process by measuring the UV-Vis absorbance and TEM images.

2.4. Synthesis of Colloidal CdTe Nanocrystals with Various OLAM : OA Ratios. All manipulations were performed using standard air-free techniques. A mixture of CdO $(0.1 \mathrm{mmol})$, OA $(0.16 \mathrm{~mL})$, and OLAM + ODE $(10 \mathrm{~mL})$ was degassed at $110^{\circ} \mathrm{C}$ for 40 minutes in a $50 \mathrm{~mL}$ three-necked flask connected to a condenser. The mixture was heated slowly under nitrogen until most of the $\mathrm{CdO}$ decomposed at $\sim 290^{\circ} \mathrm{C}$, then another $0.2 \mathrm{~mL} \mathrm{OA}$ was added to form a clear and colourless solution. A Te-TOP-TBP-ODE $(0.124 \mathrm{~mL}, \mathrm{Cd} / \mathrm{Te}=10: 7)$ precursor solution was injected quickly at $300^{\circ} \mathrm{C}$. The temperature dropped to $260^{\circ} \mathrm{C}$ and was kept at this value throughout the synthesis. Samples were taken out at different reaction times between 1 and 5 minutes.

2.5. Characterization. UV/Vis spectra were recorded using a Carry 100 absorption spectrophotometer. The samples were dispersed in $1 \mathrm{~cm}$ path length quartz cells filled with hexane. 
TABLE 1: The composition of the Te precursor solutions.

\begin{tabular}{lcccccc}
\hline Injection solutions & Te $(\mathrm{g})$ & TOP $(\mathrm{g})$ & TBP $(\mathrm{g})$ & Te solution $(\mathrm{g})$ & OLAM $\mathrm{mL}$ & ODE $\mathrm{mL}$ \\
\hline Te-TOP-OLAM & 0.5 & 4.5 & 0 & 0.0638 & 0 & 2 \\
Te-TOP-ODE & 0.5 & 4.5 & 0 & 0.0638 & 0 & 2 \\
Te-TBP-OLAM & 0.5 & 0 & 4.5 & 0.0638 & 0.0638 & 0 \\
Te-TBP-ODE & 0.5 & 0 & 4.5 & 0.1190 & 2 \\
Te-TOP-TBP-OLAM & 0.7018 & 6.5428 & 2.1054 & 0.1190 & 0 \\
Te-TOP-TBP-ODE & 0.7018 & 6.5428 & 2.1054 & & 0 \\
\hline
\end{tabular}

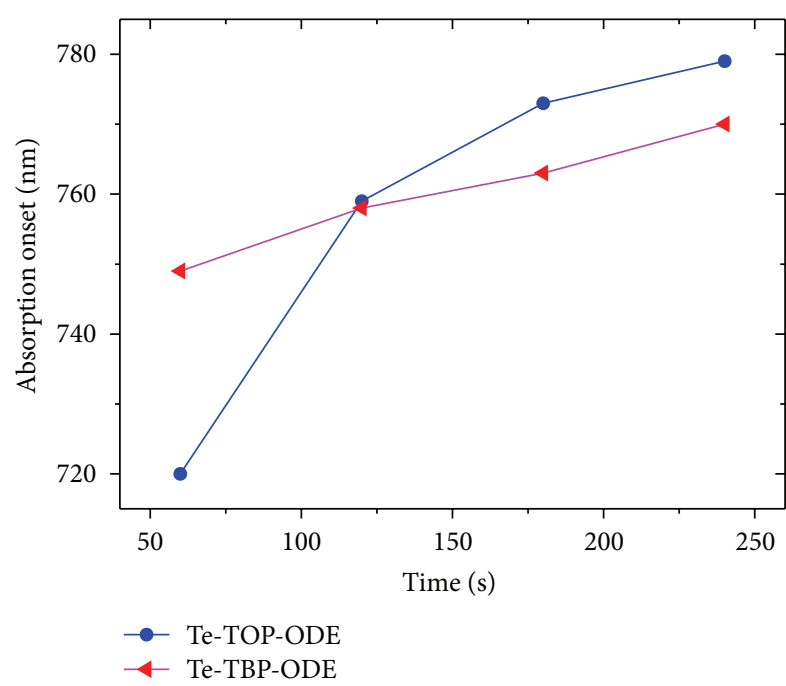

FIGURE 2: Absorption onsets of CdTe NCs synthesized with different tellurium precursor solutions: Te-TOP-ODE and Te-TBP-ODE.

The absorbance values were used to calculate the molar particle extinction coefficients, the NCs diameters, and their concentration.

2.5.1. Transmission Electron Microscopy (TEM). The size and morphology of the nanocrystals were studied with a Zeiss EM $902 \mathrm{~A}$ transmission electron microscope with an accelerating voltage of $80 \mathrm{kV}$. TEM specimens were prepared by putting a $10 \mu \mathrm{L}$ aliquot drop of a very dilute hexane solution onto a carbon-coated copper grid, which was dried in air at room temperature.

2.5.2. Powder X-Ray Diffraction (XRD). Powder X-ray diffraction (XRD) was measured with a PANalytical X'Pert PRO MPD diffractometer operating with $\mathrm{Cu} \mathrm{K} \alpha$ radiation, Bragg-Brentano $\theta-2 \theta$ geometry, a goniometer radius of $240 \mathrm{~mm}$ and variable slits. The samples were measured on low background silicon sample holders and prepared for the XRD measurements as follows. Nanocrystals in hexane solution were dropped on the silicon substrate at room temperature. The XRD data was analyzed using the X'Pert HighScore Plus software in conjunction with the ICDD database, version 2.0902 .

\section{Results and Discussion}

In this work, we investigated the influence of the reaction conditions on the shape of CdTe nanoparticles, which were synthesized by a hot-injection method in reaction of Cdoleate with trialkylphoshine telluride in OLAM as solvent. OLAM is a common organic compound which can be surfactant and solvent at the same time. OLAM reacts with OA to form an amide in the presence of metal ions $[15,17]$, which leads to a destabilization of the nanocrystals and, for example, facilitates the formation of nanowires by oriented attachment [17]. Moreover, OLAM was found to activate the metal precursors; thus, it influences the growth of nanoparticles [15, 17-19]; diluting OLAM with a noncoordinating solvent opens up the possibility to tune the activity of the metal monomers. This turned out to be an efficient way to control the shape of CdTe nanocrystals [15]. In the following, we evaluate the possibility to control the morphology of CdTe nanoparticles by additionally changing the reactivity of the Te-monomers.

3.1. Effect of Ligands and Solvents in the Tellurium Precursor Solution on the Shape of CdTe Nanocrystals. Various shapes of CdTe nanocrystals, such as nanodots, nanorods, multipods, and nanowires, can be synthesized in pure OLAM solution, only by changing the composition of the tellurium injection solution (Figure 1). Dissolving tellurium in TOP, TBP, or the mixture of them and diluting the resulting tri-alkylphosphine telluride with OLAM or ODE represent a suitable way to prepare tellurium injection solutions. TEM images of CdTe nanocrystals obtained with different Te precursor solutions are showed in Figure 1. In these six reactions we varied the ligands (TOP, TBP, or the mixture of them) and the solvent (OLAM and ODE), but we kept the Te concentration constant.

Comparison between the upper and the lower panel in Figure 1 shows that even a relatively small amount of solvent in the injection solution has a big impact on the morphology of CdTe nanocrystals (Figure 1). This can be rationalized when taking into account that amine compounds are known to activate the metal carboxylate precursors [15, 17-19]. Therefore, the ODE solvent in tellurium precursor solution decreases the activity of the Cd monomers, because it lowers the overall concentration of the amine and, thus, reduces the activation of the $\mathrm{Cd}$ monomers. All reactions with injection solutions containing OLAM (upper panel in 


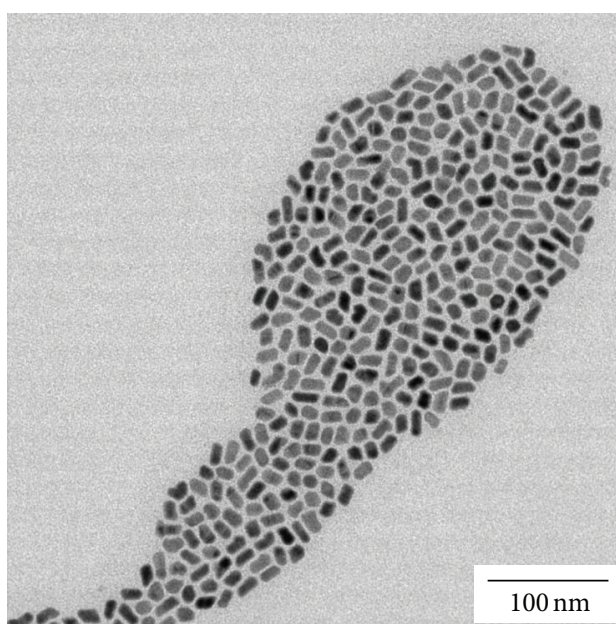

(a)

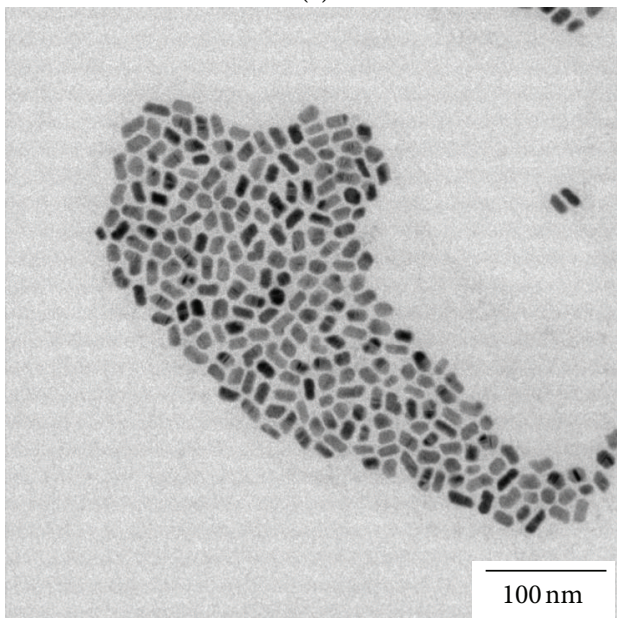

(b)

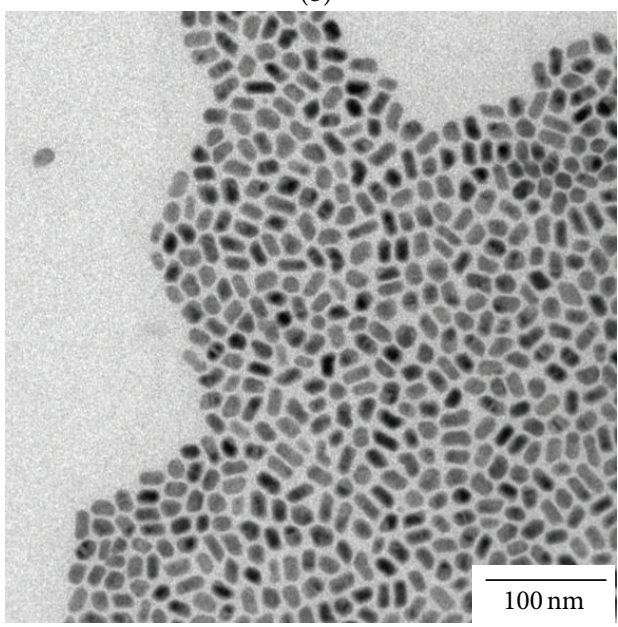

(c)

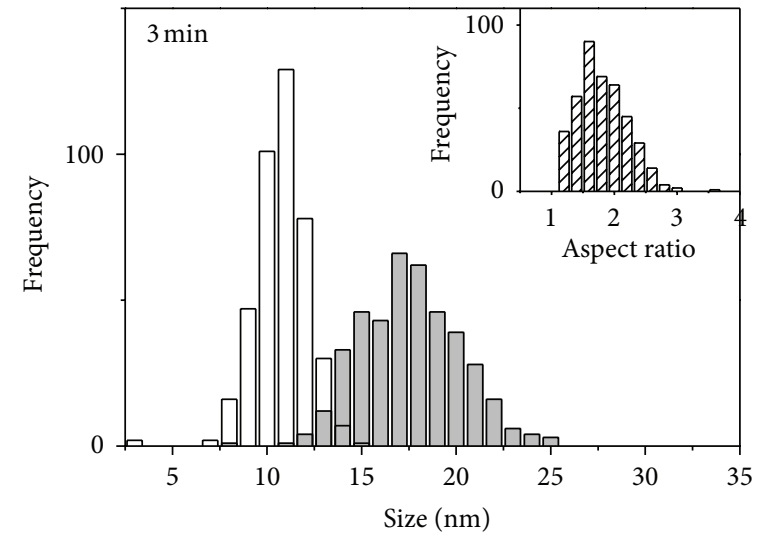

$\square$ Length

Width

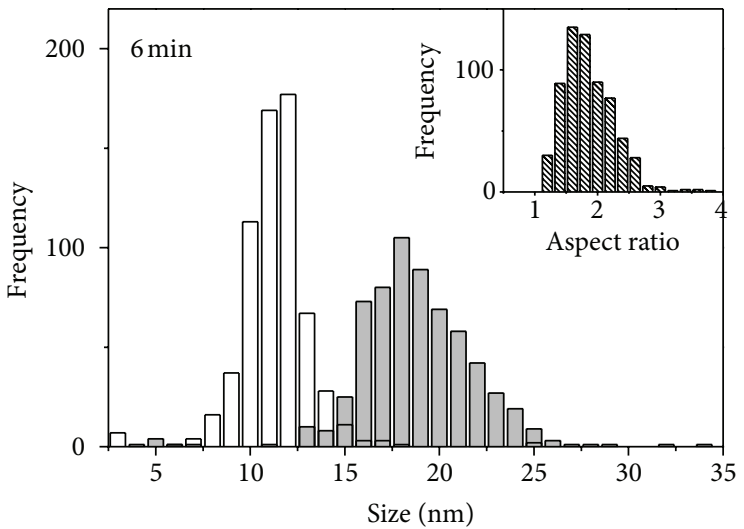

Length

Width

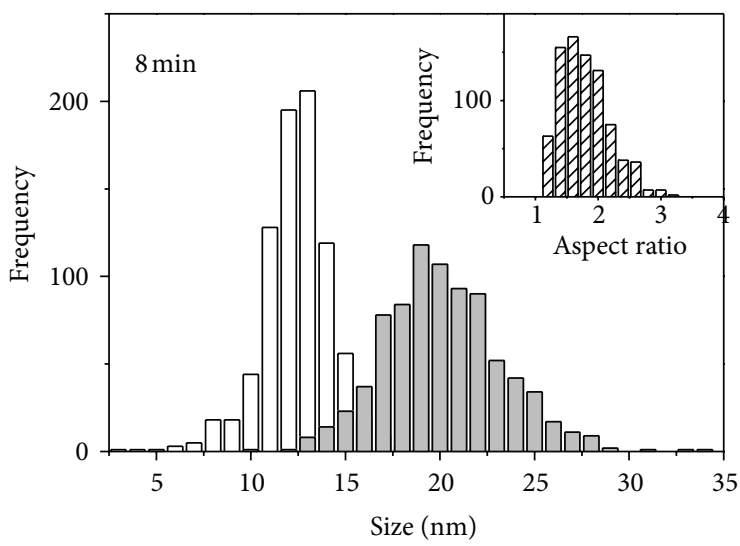

$\square$ Length

Width

Figure 3: TEM images and histogram of CdTe nanorods at different growth time. (a) 3 min; (b) 6 min; (c) 8 min.

Figure 1) yield particles with elongated shapes. This preferential one-dimensional growth is an indication for a higher monomer activity, compared with reactions with injection solutions containing ODE.
The use of TOP and TBP as ligands in tellurium precursor solutions is a good way to control the activity of the Te monomers. TOP and TBP are similar organic compounds, with $\mathrm{C} 8$ chains in TOP and C4 chains in TBP. Ligands with 


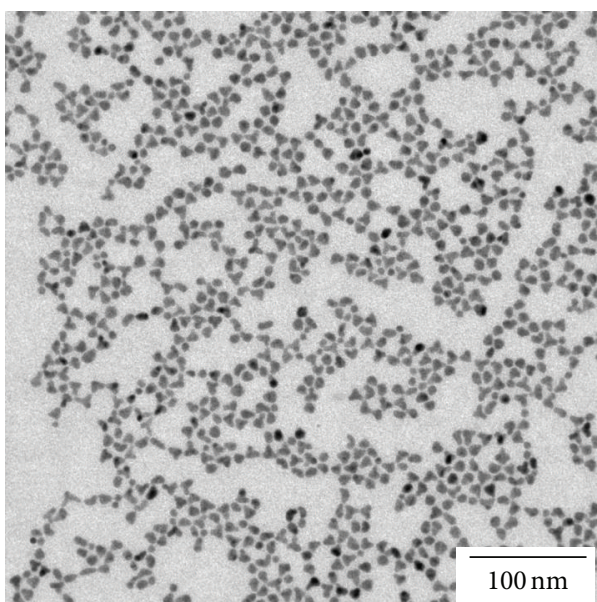

(a)

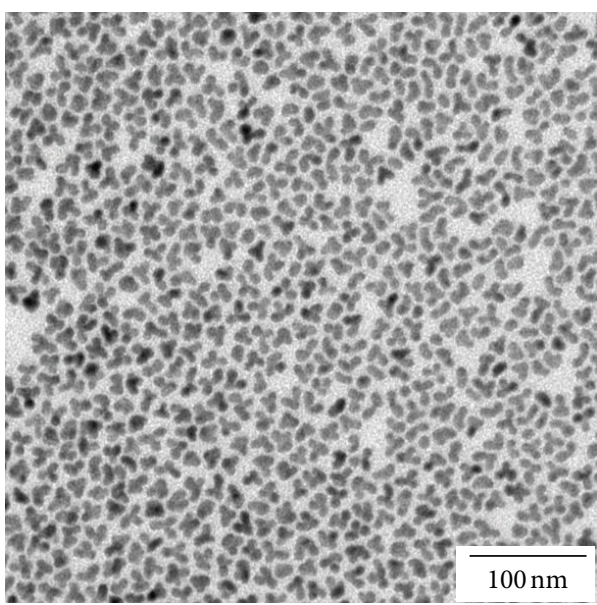

(c)

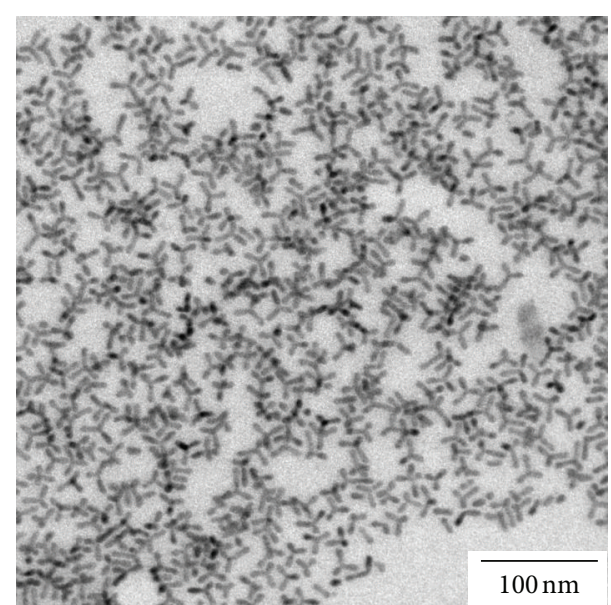

(b)

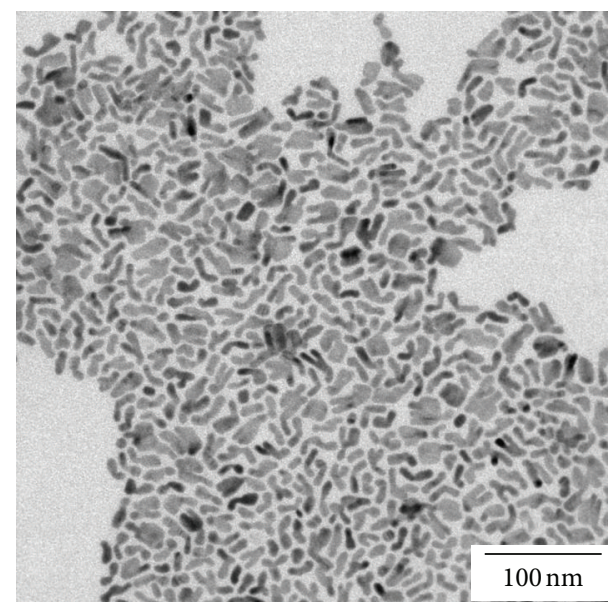

(d)

Figure 4: TEM images of CdTe NCs synthesized with different OLAM : OA ratio. (a) No OLAM; (b) OLAM: OA = 1; (c) OLAM: OA = 4; (d) OLAM: $\mathrm{OA}=20$

shorter carbon chains have a higher diffusion coefficient, which results in a higher monomer activity, compared to compounds with longer carbon chains. Furthermore, TOP$\mathrm{Te}$ and TBP-Te differ in the strength of their Te-P bonds; TOP-Te being more stable. Thus, TBP-Te is more reactive than TOP-Te. In spite of that, the results of reaction with TBP-Te and TOP-Te show some similarities; in OLAM both Te-precursors lead to the formation of nanorods, while the growth of quasispherical particles can be observed in ODE. However, a closer look at the development of the reaction shows that the underlying mechanisms are different. We compared the absorption onsets of aliquots taken during reactions with Te-TOP-ODE and Te-TBP-ODE (Figure 2). In the reaction with TBP, the particles forming during the first minute are larger than in the reaction with TOP. This is due to the higher reactivity of the Te precursor containing TBP. However, because of the formation of larger particles, a smaller amount of monomers is left for further growth. Thus, higher monomer reactivity leads to a fast monomer depletion of the solution, which slows down the growth rate of the particles at later growth stages. In the reaction with
TOP-Te, the Te precursor is less reactive, which results in the formation of smaller seeds. There are more monomers left for further growth, which is, however, slower, compared with the case of TBP-Te, because of the lower monomer activity. In the reactions of both Te precursors with more reactive $\mathrm{Cd}$ precursor (in pure OLAM) nanorods are formed, due to the relatively high chemical potential of the solution. A slightly lower Cd precursor reactivity (with ODE in the injection solution) leads to the formation of nanodots.

In reactions with Te-TOP-TBP, two Te precursors with different reactivities are present in one reaction. The more reactive Te-TBP precursor should react first and be preferentially involved in the seed formation process. The amount of monomers used up during this growth stage will be smaller, than in the case of pure Te-TBP precursor, because of the lower concentration of Te-TBP. Thus, the chemical potential of the solution remains relatively high, and also enough monomers are left for further one-dimensional growth of the nanocrystals. In the reactions with a more reactive $\mathrm{Cd}$ precursor (in pure OLAM), this leads to the formation of nanowires, while a slightly lower Cd-precursor reactivity 


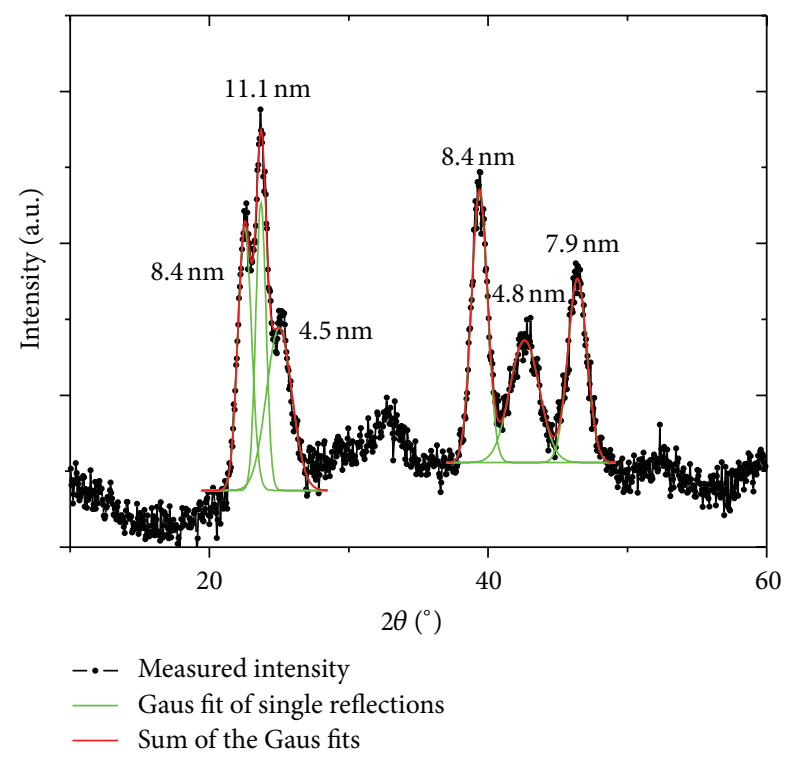

FIGURE 5: XRD pattern of CdTe NCs synthesized from OLAM : OA $=4($ TEM image in Figure 4(c)). Each reflection was fitted with a single Gaussian (green lines), and the size of the crystallites was calculated from the broadening of the reflections with the DebyeScherrer formula.

(with ODE in the injection solution) result, in the formation of tetrapods.

With TOP as ligand and OLAM as solvent in tellurium precursor solution, uniform CdTe nanorods were synthesized. Their growth was studied in more detail by TEM (Figure 3). Their mean aspect ratio is around 1.6, and it is almost not changing during the reaction (Figures $3(\mathrm{a}), 3(\mathrm{~b})$, and $3(\mathrm{c})$ ). The elongated shape forms in the beginning of the reaction and does not change during further growth. Also the size distribution of the nanorods remains the same. This shows that the monomer activity does not decrease significantly during the first 8 minutes of the reaction. In this case the shape of the nanocrystals would change from elongated to quasi-spherical; however, the monomer activity is not sufficient for the further one-dimensional growth of the nanorods.

\subsection{Morphology Control by Changing OLAM:OA Ratio.} In this section, we investigated the possibility to influence further the morphology of CdTe tetrapods. We used a TeTOP-TBP-ODE injection solution, which turned out to be suitable to synthesize uniform tetrapods (see Figure 1(e)) and changed the ratio between OLAM and OA. In these experiments, we took the same amount of OA and kept the same sum volume of OLAM and ODE constant $(10 \mathrm{~mL})$; thus, the overall CdTe concentration was fixed. In this way we could exclude that the effects we observe are solely due to changes in the concentration of the reactants.

Figure 4 shows the different morphologies of CdTe nanocrystals obtained by changing OLAM: OA ratio from 0 to 20. In our experiment, the use of OA as the only surfactant leads to the formation of triangular (in the twodimensional projection) nanoparticles (Figure 4(a)). Without OLAM the reactivity of the Cd-monomers is low (no amine activation); thus, there is no one-dimensional growth. The presence of the same volume of OLAM and OA in the reaction system causes the formation of tripods and tetrapods (Figure 4(b)). With larger amount of OLAM, the thickness of the arms of the tetrapods increases, while their length decreases (Figure 4(c)). With even higher OLAM concentrations, formation of more irregular shapes of branched CdTe nanocrystals, such as Y-shaped multipods, can be observed. Also the fraction of flat, two-dimensional CdTe nanoparticles increases with higher OLAM concentrations (Figure 4(d)). Thus, the presence of OLAM influences the growth direction of the CdTe nanocrystals.

Figure 5 shows the XRD patterns of CdTe nanocrystals shown in Figure 4(c). Each reflection was fitted with a single Gaussian function (green curves in Figure 5); the sum of the fitting curves, which corresponds well to the measured diffraction pattern, is shown as red lines in Figure 5. The diffraction peaks can be assigned to wurtzite CdTe (ICDD number 01-073-2871). Results of the calculation of the crystallite dimension in the corresponding directions using the Scherrer equation with $K=1$ for undefined geometry are included in Figure 5. Because of the anisotropic growth of the nanocrystals, we obtain different values for different reflections. The relatively sharp (002) peak in the diffraction pattern is consistent with the preferential growth of the CdTe NCs in the [001] direction. The crystallite size in the [001] direction $(11.1 \mathrm{~nm})$ corresponds well to the length of the arms of the tetrapods measured from the TEM images.

\section{Conclusions}

In conclusion, we demonstrated that various shapes of CdTe nanocrystals could be synthesized by controlling the composition of the solvent and of the tellurium precursor solution. Tuning the reactivity of both the cadmium and the tellurium precursors is the main reason for the shape control of $\mathrm{CdTe}$ nanocrystals in such a simple system. The activity of the $\mathrm{Cd}$ precursor can be controlled by the concentration of OLAM in the solvent, while the use of TOP, TBP, or a mixture of both phosphines in the tellurium injection solution turned out to be a suitable way to control the reactivity of the Te monomers. Changing the reactivity of the $\mathrm{Cd}$ and $\mathrm{Te}$ precursors at the same time opens up the possibility to influence the seed formation and the growth process of the CdTe nanocrystals and, thus, to control their shape.

\section{Acknowledgments}

The authors would like to thank Erhard Rhiel and Heike Oetting (University of Oldenburg) for assistance in obtaining TEM images and Marta Kruszynska (University of Oldenburg) for taking the XRD data. They gratefully acknowledge funding of the EWE Research Group "Thin Film Photovoltaics" by the EWE AG, Oldenburg. 


\section{References}

[1] A. I. Hochbaum and P. Yang, "Semiconductor nanowires for energy conversion," Chemical Reviews, vol. 110, no. 1, pp. 527$546,2010$.

[2] P. V. Kamat, "Quantum dot solar cells. Semiconductor nanocrystals as light harvesters," The Journal of Physical Chemistry C, vol. 112, pp. 18737-18753, 2008.

[3] Q. Ma and X. Su, "Near-infrared quantum dots: synthesis, functionalization and analytical applications," The Analyst, vol. 135, no. 8, pp. 1867-1877, 2010.

[4] P. Alivisatos, "The use of nanocrystals in biological detection," Nature Biotechnology, vol. 22, no. 1, pp. 47-52, 2004.

[5] Z. Tang, N. A. Kotov, and M. Giersig, "Spontaneous organization of single CdTe nanoparticles into luminescent nanowires," Science, vol. 297, no. 5579, pp. 237-240, 2002.

[6] Z. Tang, Z. Zhang, Y. Wang, S. C. Glotzer, and N. A. Kotov, "Self-assembly of CdTe nanocrystals into free-floating sheets," Science, vol. 314, no. 5797, pp. 274-278, 2006.

[7] H. Qian, C. Dong, J. Weng, and J. Ren, "Facile one-pot synthesis of luminescent, water-soluble, and biocompatible glutathionecoated CdTe nanocrystals," Small, vol. 2, no. 6, pp. 747-751, 2006.

[8] Y. A. Yang, H. Wu, K. R. Williams, and Y. C. Cao, "Synthesis of CdSe and CdTe nanocrystals without precursor injection," Angewandte Chemie-International Edition, vol. 44, no. 41, pp. 6712-6715, 2005.

[9] H. Zhang, D. Wang, and H. Möhwald, "Ligand-selective aqueous synthesis of one-dimensional CdTe nanostructures," Angewandte Chemie-International Edition, vol. 45, no. 5, pp. 748-751, 2006.

[10] J. Y. Zhang and W. W. Yu, "Formation of CdTe nanostructures with dot, rod, and tetrapod shapes," Applied Physics Letters, vol. 89, no. 12, Article ID 123108, 2006.

[11] R. Zheng, S. Guo, and S. Dong, "Synthesis of CdTe nanocrystals using Te nanorods as the Te source and the formation of microtubes with red fluorescence," Inorganic Chemistry, vol. 46, no. 17, pp. 6920-6923, 2007.

[12] W. W. Yu, Y. A. Wang, and X. Peng, "Formation and stability of size-, shape-, and structure-controlled CdTe nanocrystals: ligand effects on monomers and nanocrystals," Chemistry of Materials, vol. 15, no. 22, pp. 4300-4308, 2003.

[13] J. Kolny-Olesiak, V. Kloper, R. Osovsky, A. Sashchiuk, and E. Lifshitz, "Synthesis and characterization of brightly photoluminescent CdTe nanocrystals," Surface Science, vol. 601, no. 13, pp. 2667-2670, 2007.

[14] V. Kloper, R. Osovsky, J. Kolny-Olesiak, A. Sashchiuk, and E. Lifshitz, "The growth of colloidal cadmium telluride nanocrystal quantum dots in the presence of Cd0 nanoparticles," Journal of Physical Chemistry C, vol. 111, no. 28, pp. 10336-10341, 2007.

[15] X. Jin, J. Li, J. Parisi, and J. Kolny-Olesiak, "Shape control of CdTe nanocrystals synthesized in presence of in situ formed CdO particles," Journal of Nanoparticle Research, vol. 13, no. 12, pp. 6963-6970, 2011.

[16] J. W. Cho, H. S. Kim, Y. J. Kim et al., "Phase-tuned tetrapodshaped CdTe nanocrystals by ligand effect," Chemistry of Materials, vol. 20, no. 17, pp. 5600-5609, 2008.

[17] X. Jin, M. Kruszynska, J. Parisi, and J. Kolny-Olesiak, "Catalystfree synthesis and shape control of CdTe nanowires," Nano Research, vol. 4, no. 9, pp. 824-835, 2011.
[18] L. S. Li, N. Pradhan, Y. Wang, and X. Peng, "High quality ZnSe and $\mathrm{ZnS}$ nanocrystals formed by activating zinc carboxylate precursors," Nano Letters, vol. 4, no. 11, pp. 2261-2264, 2004.

[19] N. Pradhan and X. Peng, "Efficient and color-tunable Mndoped ZnSe nanocrystal emitters: control of optical performance via greener synthetic chemistry," Journal of the American Chemical Society, vol. 129, no. 11, pp. 3339-3347, 2007. 

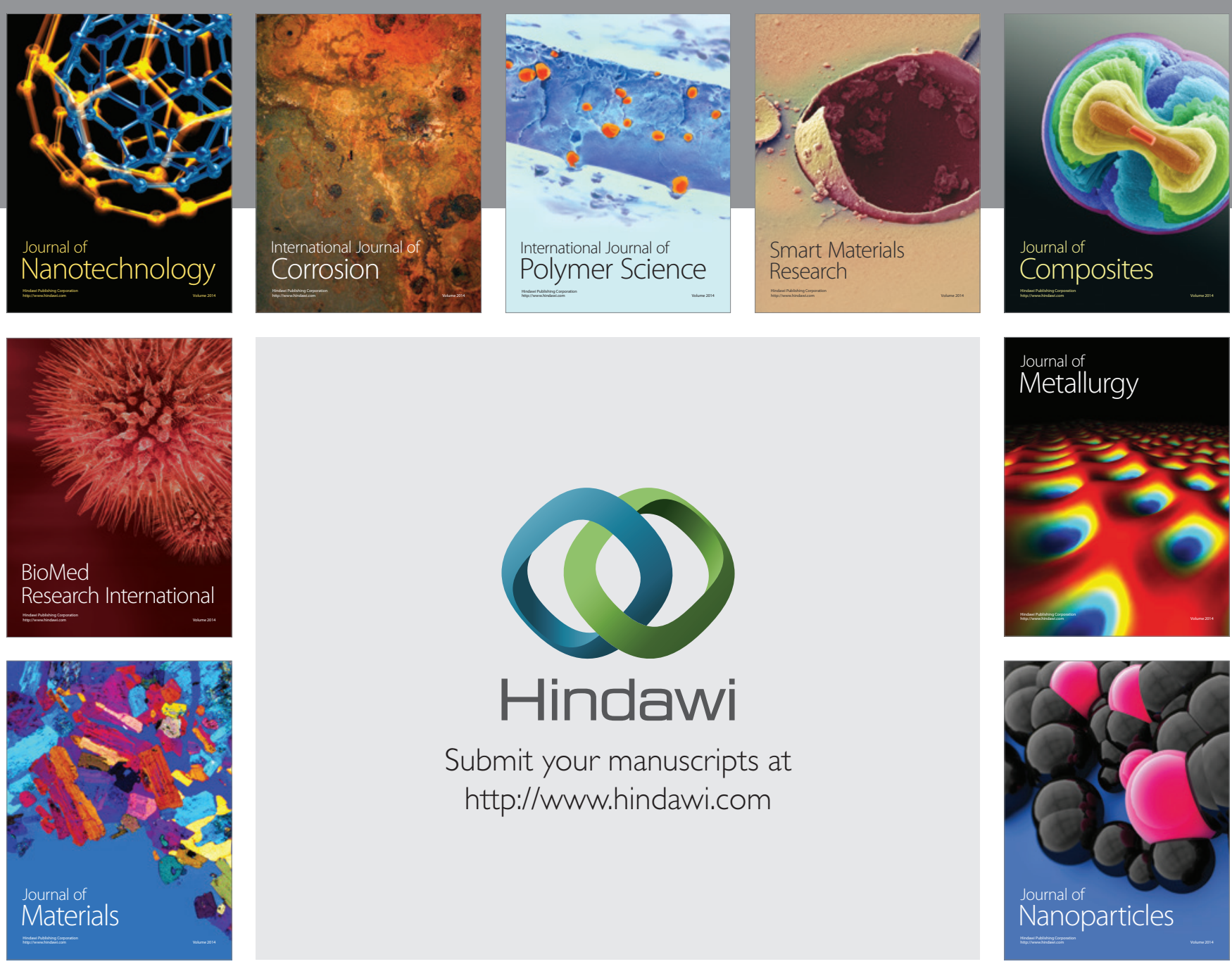

Submit your manuscripts at http://www.hindawi.com
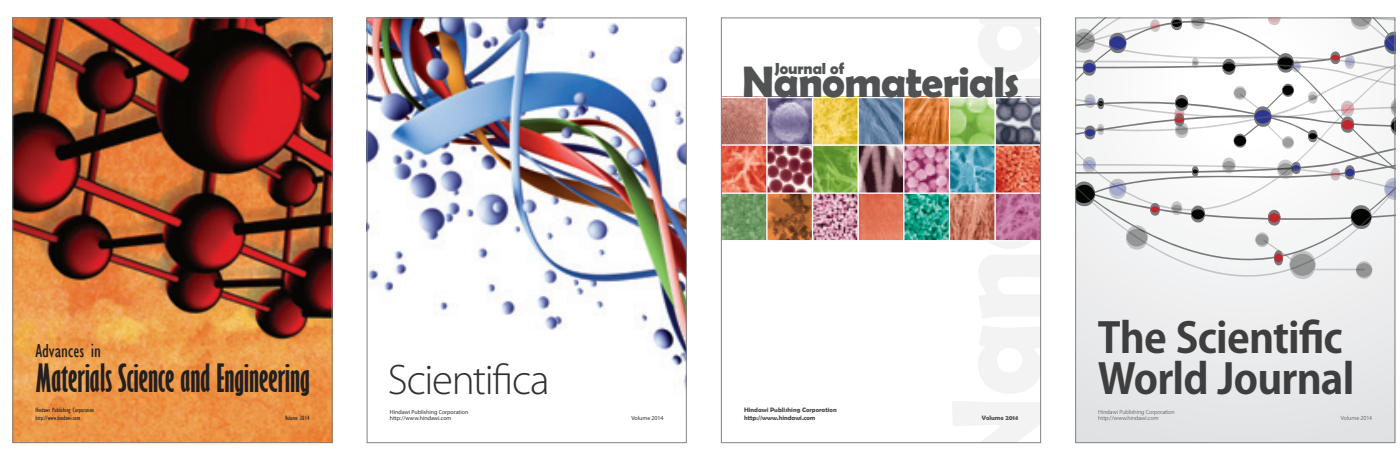

\section{The Scientific World Journal}
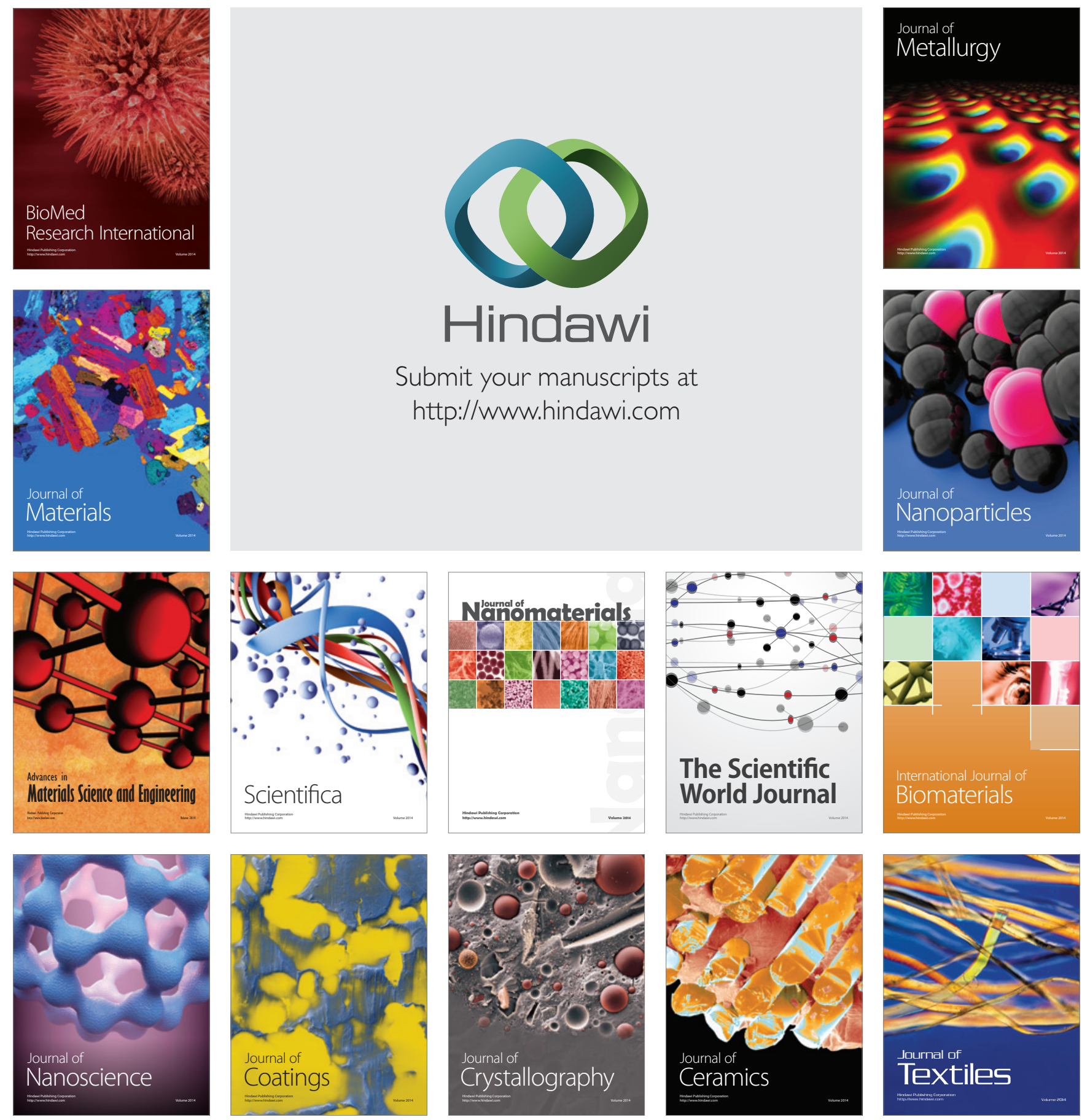